Article

\title{
Integrated Laser Sensor (ILS) for Remote Surface Analysis: Application for Detecting Explosives in Fingerprints
}

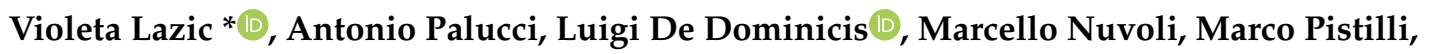 \\ Ivano Menicucci, Francesco Colao and Salvatore Almaviva \\ Energy and Sustainable Economic Development (ENEA), Italian National Agency for New Technologies, \\ FSN-TECFIS-DIM, Via E. Fermi 45, 00044 Frascati (RM), Italy; antonio.palucci@enea.it (A.P.); \\ luigi.dedominicis@enea.it (L.D.D.); marcello.nuvoli@enea.it (M.N.); marco.pistilli@enea.it (M.P.); \\ ivano.menicucci@enea.it (I.M.); francesco.colao@enea.it (F.C.); salvatore.almaviva@enea.it (S.A.) \\ * Correspondence: violeta.lazic@enea.it; Tel.: +39-06-9400-5885
}

Received: 29 August 2019; Accepted: 27 September 2019; Published: 1 October 2019

check for updates

\begin{abstract}
Here, we describe an innovative Integrated Laser Sensor (ILS) that combines four spectroscopic techniques and two vision systems into a unique, transportable device. The instrument performs Raman and Laser-Induced Fluorescence (LIF) spectroscopy excited at $355 \mathrm{~nm}$ and Laser-Induced Breakdown Spectroscopy (LIBS) excited at $1064 \mathrm{~nm}$, and it also detects Laser Scattering (LS) from the target under illumination at $650 \mathrm{~nm}$. The combination of these techniques supplies information about: material change from one scanning point to another, the presence of surface contaminants, the molecular and elemental composition of top target layers. Switching between the spectroscopic techniques and the laser wavelengths is fully automatic. The instrument is equipped with an autofocus and it performs scanning with a chosen grid density over an interactively-selected target area. Alternative to the spectroscopic measurements, it is possible to switch the instrument to a high magnification target viewing. The working distances tested until now are between 8.5 and $30 \mathrm{~m}$. The instrument is self-powered and remotely controlled via wireless communication. The ILS has been fully developed at ENEA for security applications and it was successfully tested in two outdoor campaigns where an automatic recognition of areas containing explosives in traces had been implemented. The strategies for the identification of nitro-compounds placed on various substrates as fingerprints and the results obtained at a working distance of $10 \mathrm{~m}$ are discussed in the following.
\end{abstract}

Keywords: LIBS; Raman; LIF; stand-off; explosives; fingerprints; scanning; laser spectroscopy; laser scattering; residue; remote

\section{Introduction}

Remote measurements of the atomic and molecular composition of solid samples have a wide range of applications, among them are the analysis of pigments in cultural heritage, detection of explosives and their precursors, analysis of geological samples, measurements for forensics, space exploration, and industrial and environmental monitoring. The most-used techniques for remote molecular analysis of solids are Raman [1-5] and Laser-Induced Fluorescence (LIF) [6-9] while Laser-Induced Breakdown Spectroscopy (LIBS) supplies information about the elemental composition of the environment [10] or of the top target layers [11-16].

The three spectroscopic methods (Raman, LIF, and LIBS) provide complementary information and they might have some common components like a laser source, beam focusing, a signal collection system, as well as spectrometers. These facts have prompted an interest and several efforts to develop 
a sensing device that integrates LIBS and Raman [17-20] or all three techniques, as discussed in the following. Osticcioli et. al. [21] built a combined instrument for proxy (working distance of $2.5 \mathrm{~cm}$ ) characterization of objects related to cultural heritage. They switched manually a single laser source between $532 \mathrm{~nm}$ for Raman and LIBS and $266 \mathrm{~nm}$ for LIF, then inserted or removed various filter and changed by PC commands the monochromator gratings to get High Resolution (HR) Raman spectra or lower resolution LIBS and LIF spectra in a larger spectral interval.

The first laboratory instrument for remote sensing by the three techniques was described in [22], aimed also at planetary exploration. This combined instrument contains a single laser source emitting at $532 \mathrm{~nm}$ and a single detector covering the spectral range of 535-680 nm. The Raman signal can be partially separated from LIF emission by restricting the acquisition gate in the proximity of the laser pulse arrival. The LIBS measurements with the described system suffer from a limited detected spectral range where the only lines observed from a rock material belong to $\mathrm{Fe}, \mathrm{Na}, \mathrm{Ca}$, and $\mathrm{Li}$.

The most advanced Raman-LIF-LIBS device reported until now regards the SuperCam instrument, intended to be mounted on a rover and used by NASA for the Mars 2020 mission [23,24]. The SuperCam integrates many remotely-controlled instruments, among them there is LIBS excited at $1064 \mathrm{~nm}$ plus Raman/LIF generated at $532 \mathrm{~nm}$, with the measuring range up to $7 \mathrm{~m}$ and $12 \mathrm{~m}$, respectively. The instrument is equipped with different spectrometer channels covering certain ranges in UV, VIS, and NIR, thus providing a large number of elements detectable by LIBS.

In the present paper we describe a patent-pending ENEA system ILS for remote material characterization by Raman, LIF, LIBS, and LS techniques. The instrument is equipped with two vision systems, it is fully automatized and has also a scanning capability. Although the ILS might have many applications, some of them were already tested like forensics, characterization of rocks or objects related to cultural heritage, etc., here we describe the system itself and show the preliminary results on explosives in traces, the detection of which still remains a challenging issue $[2-5,7,15,18,25]$, particularly on remote targets [26]. The contaminants were left on substrates as fingerprints, which are considered as the most important mechanisms for the transfer of trace amounts of explosives during bomb handling and preparation [27].

\section{Experimental}

\subsection{Overview}

The ILS instrument was initially developed as a laboratory prototype with separate controls for each device and with manual movement of the optical components mounted on micrometric slits. After optimization of the optical lay-out, the components were integrated into a fully-automatized Prototype 2 (Figure 1), controlled remotely by home-developed software. This system had been successfully tested at the end of 2015 during one demo campaign. Next, we built Prototype 3, where the mechanical stability was improved by integrating the laser head into the rotating platform (Figure 2). In the following, we describe and discuss the last instrument version (Prototype 3 ).

The ILS consists of the instrument box and the platform rotating both horizontally and azimuthally. The rotating platform carries the optical head of a high-power laser, the diode laser for measuring the target distance, two video cameras, optical components for launching the laser beams, then the telescope for the signal collection, and behind it, the multiplexer box for signal delivery. The instrument box encloses two spectrometers, various power supplies, control electronics, the motors of the scanning system, an air-cooling system, a PC server, and a WiFi transmitter/receiver. The two units are connected electrically via an umbilical cable while the two optical fibers deliver the signal to the respective spectrometers. The overall system specifications are given in Table 1.

The LS measurements here are intrinsically eye-safe while LIF and Raman spectroscopy could be performed in eye-safe regime by limiting the laser energy and number of pulses. For example, according to the ANSI Standard, in eye-safe measurements with only one delivered ns laser pulse at $355 \mathrm{~mm}$, the maximum permissible exposure is $5.6 \mathrm{~mJ} / \mathrm{cm}^{2}$. 


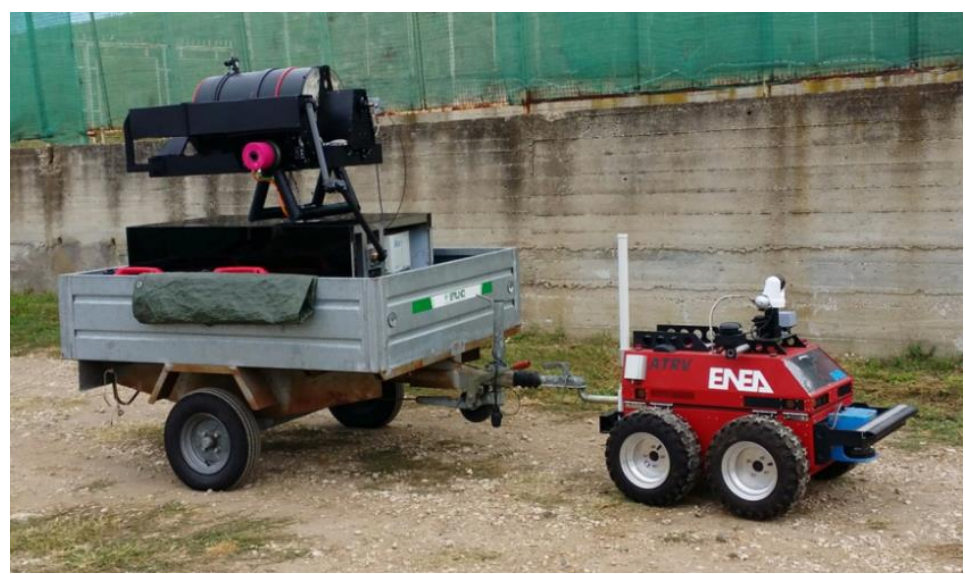

Figure 1. Integrated Laser Sensor (ILS) Prototype 2 placed on a trailer and driven by a remotely-operated vehicle.
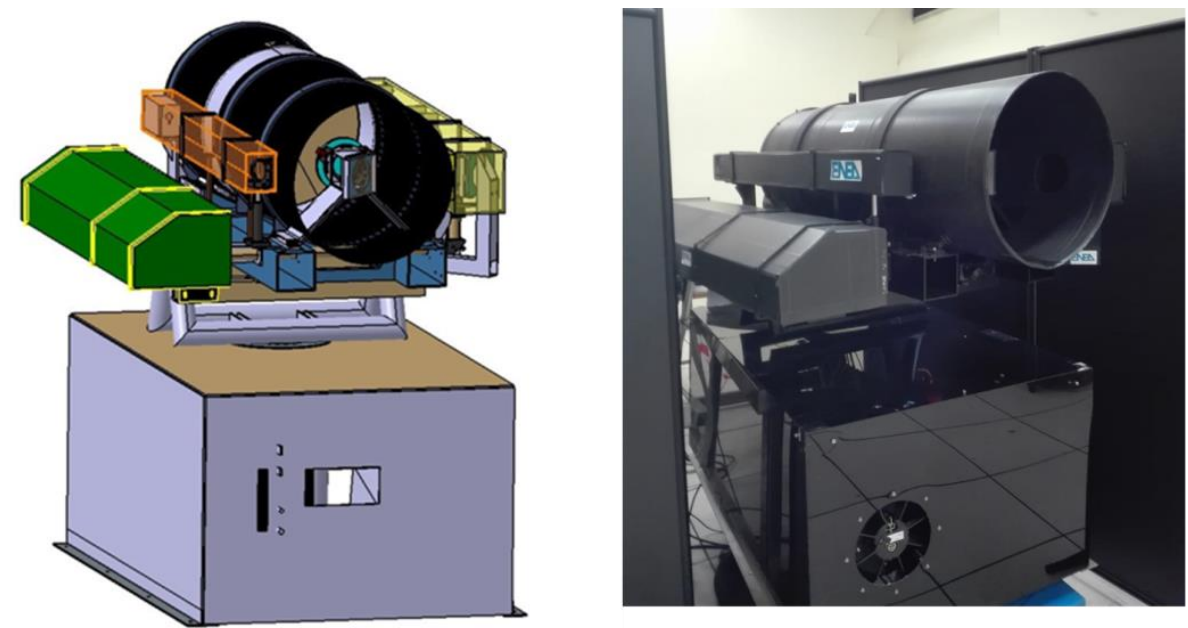

Figure 2. Drawing (left) and photo (right) of the ILS Prototype 3.

Table 1. Specification of the ILS system.

\begin{tabular}{|c|c|c|}
\hline Feature & Specifications & Notes \\
\hline Distance measurements & Limit $500 \mathrm{~m}^{(1)}$, resolution $0.1 \mathrm{~mm}$ & (1) On reflective target \\
\hline Target distance & $8.5-30 \mathrm{~m}^{(2)}$ & (2) tested; estimated >100 m \\
\hline Autofocus & Yes & \\
\hline Scanning resolution & $0.1 \mathrm{mrad}$ & $1 \mathrm{~mm}$ at a distance of $10 \mathrm{~m}$ \\
\hline LIF measurements & $\begin{array}{c}\text { Laser@355 nm, filter }{ }^{(3)} \text {, Echelle } \\
\text { spectrometer }\end{array}$ & (3) Razor edge $355 \mathrm{~nm}$ \\
\hline Scattering measurements & $\begin{array}{l}\text { Laser @650 nm, filter }{ }^{(3)} \text {, Echelle } \\
\text { spectrometer }\end{array}$ & Simultaneous with LIF \\
\hline Raman measurements & $\begin{array}{l}\text { Laser @355 nm, filter }{ }^{(3)} \text {, } \\
\text { Czerny-Turner }^{(4)}\end{array}$ & (4) Higher throughput than Echelle \\
\hline LIBS measurements & $\begin{array}{c}\text { Laser @1064 nm, Echelle } \\
\text { spectrometer }\end{array}$ & \\
\hline $\begin{array}{l}\text { Switching time between two } \\
\text { measuring techniques }\end{array}$ & $10 \mathrm{~s}^{(5)}$ & (5) Limited by the software \\
\hline External camera & $\begin{array}{c}\text { Color, } 1280 \times 1024 \text { pixel, with } \\
\text { objective f } 35\end{array}$ & Always acquiring \\
\hline
\end{tabular}


Table 1. Cont.

\begin{tabular}{ccc}
\hline Feature & Specifications & Notes \\
\hline Internal camera & $\begin{array}{c}\text { Color, } 1280 \times 1024 \text { pixel, } \\
\text { magnification } 3.5 \mathrm{X}-22 \times \text { for the } \\
\text { target distance of } 8.5-30 \mathrm{~m}\end{array}$ & $\begin{array}{c}\text { Alternative to the spectroscopic } \\
\text { measurements }\end{array}$ \\
\hline Control & WiFi and the dedicated software ${ }^{(6)}$ & ${ }^{(6)}$ ENEA's property \\
\hline Ambient temperature & $10{ }^{\circ} \mathrm{C}(7)-35^{\circ} \mathrm{C}$ & ${ }^{(7)}$ tested $10{ }^{\circ} \mathrm{C}-40{ }^{\circ} \mathrm{C}$ \\
\hline
\end{tabular}

\subsection{Optical System}

The optical system of the ILS is schematically drawn in Figure 3. The high-power laser (Q-smart 850, Quantel, Newbury, UK) emitted 6 ns-long pulses at a repetition rate up to $10 \mathrm{~Hz}$ and with a maximum energy of $850 \mathrm{~mJ}$ and $230 \mathrm{~mJ}$ at wavelengths of $1064 \mathrm{~nm}$ and $355 \mathrm{~nm}$, respectively. Before and after the frequency conversion unit, there is a motorized slit carrying two laser mirrors each, used to bypass the nonlinear crystals and so delivering the beam at fundamental harmonics for LIBS measurements (not shown). Alternatively, the mirrors do not intercept the laser beam exiting the resonator, so the fundamental beam is converted to $355 \mathrm{~nm}$ and used for Raman and LIF spectroscopy. Both the UV and the IR beam are centered at the exit aperture of the laser head unit and aligned angularly to follow the same optical path.

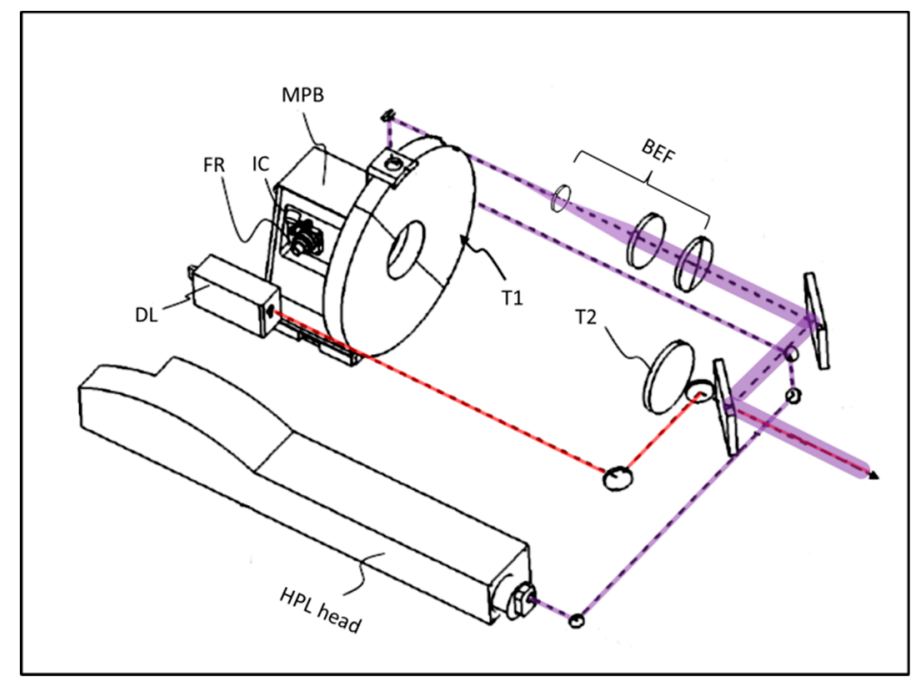

Figure 3. A simplified drawing of the overall optical system where DL is the distance-meter, T1 and T2 are telescope mirrors, MPB is the multiplexer box, FR is the fiber attachment for Raman, and IC is the internal camera. The violet thick line indicates the high-power laser beam, while the red line indicates the beam from the DL.

In order to reduce the length of the rotating unit, the high-power laser beam exiting the laser head is bent by mirrors (HR 1064/355) and brought to the back side of the platform. The laser radiation then passes through the beam expander/focuser, the last lens of which has a diameter of $100 \mathrm{~mm}$. The first lens of this optical unit is mounted on a motorized slit in order to adjust the focus according to the target distance and the laser wavelength. The expanded high-power laser beam is injected into the telescope's axis by means of two dielectrically-coated mirrors that are transparent to radiation of the laser diode $(650 \mathrm{~nm})$ emitted by the distance-meter (DIMETIX, FLS-C10 $\mathrm{nm}$ ). The red beam is brought along the telescope's axis by two metallic mirrors, and the second one is placed behind the last mirror for the high-power laser beam.

The optical signal from the target is collected and focused by a Cassagrain telescope, the primary mirror of which has a diameter of $400 \mathrm{~mm}$. Both telescope mirrors are coated with AlMgF in order to 
maximize the reflectivity in the UV spectral region. The primary mirror, together with the multiplexer box, is mounted on a motorized stage that keeps the target in focus when the working distance changed. The multiplexer box distributes the optical signal to one of the two optical fibers or to the internal camera, and it also inserts/removes the razor edge filter that cuts the UV laser radiation (not shown).

LIBS and LIF spectra are detected by the Echelle spectrometer (Mechelle 5000, Andor, Belfast, UK) equipped with an ICCD (iStar DH334T, Andor), covering the range of 200-900 nm. The quartz fiber connecting this unit with the multiplexer has an $800-\mu \mathrm{m}$ core diameter. For collecting the intrinsically-weak Raman signal, we use a bundle containing $100-\mu \mathrm{m}$ diameter fibers arranged at the exit into an array to match the slit of the Czerny-Turner spectrometer (iHR-320, HORIBA, Kyoto, Japan) equipped with a CCD detector (iKon-M934, Andor).

Due to the large achievable laser beam expansion (up to $10 \times$ ) the minimum theoretical spot size on a target, calculated for the beam quality $\mathrm{M}^{2}=2$, is relatively small (Table 2). However, for LIBS, we consider the optimal spot size as a value corresponding to the maximum plasma emission that occurrs when focusing the beam beyond the target surface. In order to collect as much Raman signal as possible, for this technique, the high-power laser beam is defocused to match the diameter of the target area imaged by the telescope on the fiber bundle. For simplicity, the same laser spot size is used for LIF, although here and in the LS measurements, the signal is collected from a smaller area, corresponding to the image of the single, large core fiber. The lateral resolution of the measurements is given in Table 2, and it could be improved in Raman and LIF measurements by a tighter focusing of the high-power laser beam.

Table 2. The minimum theoretical laser spot size for three target distances, the used laser spot size, and the lateral resolution for Raman, LIF and LIBS measurements.

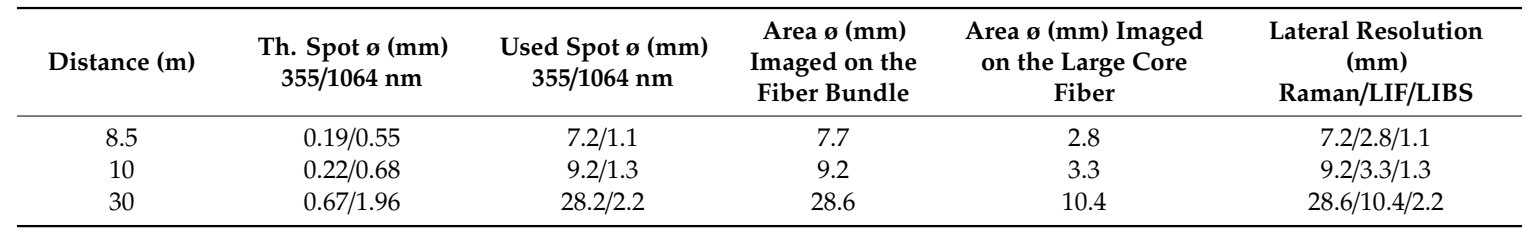

\subsection{Software}

Software for control of the ILS instrument was developed in a Net.4 Windows environment. The program core activates and controls all the devices, which correct functioning allows launching the interactive interface to perform the spectroscopic measurements. The user's interface on the desktop, photographed after target scanning by Raman technique, is shown in Figure 4. The central image is continuously taken by the external camera.

The signal processing routines, like background subtraction and de-noising of the Raman spectra, as well as peak identification to classify nitro-compounds starting from the built-in database, were written in LabView and recalled by the main program. The analogue routines were written for recognition of a generic nitro-compound by LIBS. For both techniques, the areas where a nitro-compound was detected appear in real-time as red cells on the target image. In the case of Raman measurements, the name of the identified nitro-compound and the corresponding measured spectrum appear on the desktop if clicking on the specific target's point.

The internal camera is used only in specific cases, and it is presently controlled out of the main instrument's program. 


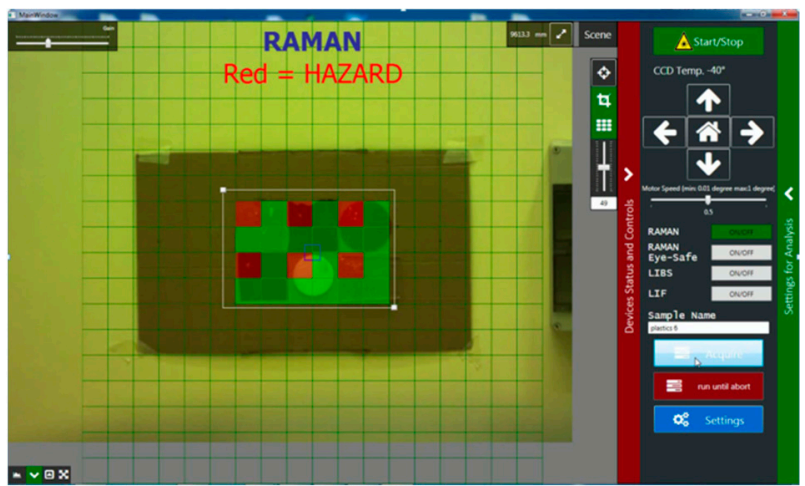

Figure 4. User interface photographed after Raman scanning of six plastic materials, each one containing an ammonium-nitrate fingerprint in the upper left corner. The largest area of the desktop is occupied by the image (video) acquired by the external camera. Fingerprints containing ammonium-nitrate were identified in real time, producing a red-colored cell in correspondence with its position on the target.

\subsection{Materials and Methods}

For the first outdoor campaign, the task was to identify fingerprints of nitro-compounds left on a vehicle. In preliminary laboratory measurements, we placed, with a silicon finger, residues up to the 10th generation on black polypropylene from the internal car and on white varnished metal from the external car. The analyzed residues included different military-grade explosives: trinitrotoluene (TNT), cyclotrimethylene-trinitramine (RDX), cyclotetramethylene-tetranitramine (HMX), and pentaerythritol-tetranitrate (PETN), plus their possible precursors as analytical-grade ammonium-nitrate and urea-nitrate, plus some common interferents like dust, wax, hand-cream, diesel, lubricating oil, and grease. Except for the LIBS, performed at 1-4 points per fingerprint, the measurements regarded the central part of fingerprint left on the substrate. Furthermore, inside the second outdoor campaign, we tested a limited number of fingerprints per substance on other substrates like textile, jeans, card, and some other plastic materials.

\section{Results}

In the multi-technique target scanning by the ILS, we usually apply the following sequence:

1. LIF and LS measurements for identifying eventual changes of the target material (roughness and surface contamination).

2. Eventual inspection of the previously-tagged interesting areas by the internal camera.

3. Raman measurements over the whole target or just over the previously-tagged areas, in order to obtain eventual molecular identification.

4. At the end, we performe LIBS measurements (micro-destructive) that supplies information about the elemental composition of the top sample layer(s).

\subsection{LIF and LS Measurements}

The LIF measurements were performed at a target distance of $10 \mathrm{~m}$ by applying the laser energy of $16 \mathrm{~mJ}$ and the signal accumulation over 10 laser pulses. The acquisition was pre-triggered $500 \mathrm{~ns}$ before the high-power laser pulse, and the gate width was set to $0.1 \mathrm{~ms}$ as a compromise to capture both the expected short-living LIF signal and the scattered light under continuous-wave illumination by the red laser, without masking the signals by the ambient light. Examples of LIF + LS processed spectra (corrected for the spectral response and smoothed over 10 point) from black polypropylene and from white rough nylon containing clean areas or first-generation fingerprints of different contaminants, are shown in Figure 5. On the black polypropylene, we observed fluorescence from ammonium-nitrate, having a wide peak around $460 \mathrm{~nm}$, together with its Raman peak $\left(368.3 \mathrm{~nm}\right.$ or $\left.1044 \mathrm{~cm}^{-1}\right)$. The grease 
residue had a very intense LIF signal peak around $420 \mathrm{~nm}$. On the same black target we observed a wide LIF peak centered on $530 \mathrm{~nm}$ coming from all the examined nitro-compounds except for PETN. All the tested organic interferences had the LIF peak closer to the laser excitation wavelength (not shown). The detected LS signal increased in the presence of powder residues compared to the clean substrate while it dropped on a greasy area. We detected the differences in LS and LIF signals between the black clean substrate and all the examined fingerprints on the black substrate.
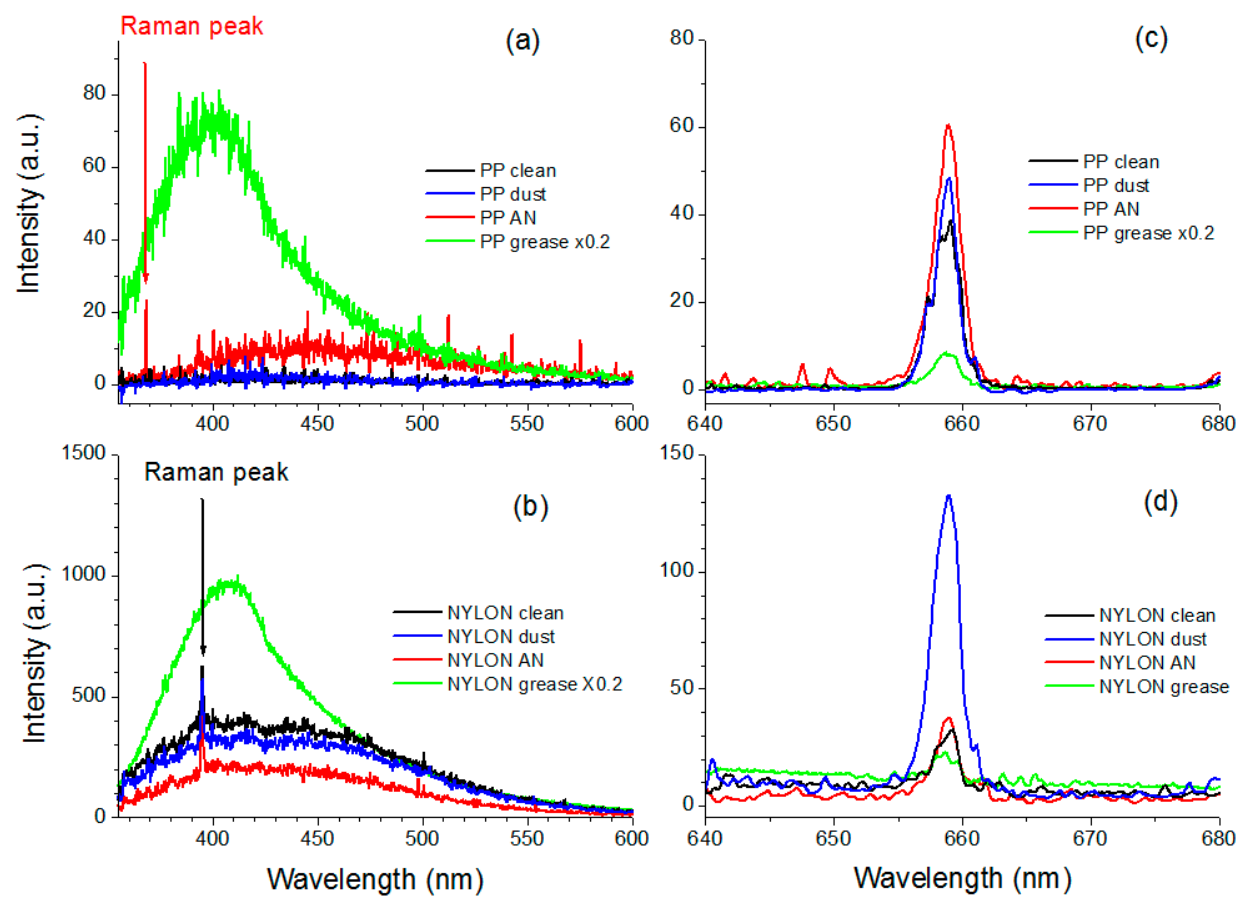

Figure 5. LIF spectra from polypropylene (PP) (a) and nylon (b) measured on clean area and on fingerprints containing ammonium-nitrate (AN), dust, or grease. The corresponding LS peaks are shown in the right panels $(\mathbf{c}, \mathbf{d})$.

Differently, on the fluorescing nylon target also the Raman peak due to C-H bond ( $395.2 \mathrm{~nm}$ or $2900 \mathrm{~cm}^{-1}$ ) was observed and the examined powdered residues decreased the LIF signal intensity, i.e., they partially masked the substrate's fluorescence. In this case as well, the greasy residues had an intense fluorescence, the peak of which was shifted towards the UV compared to the clean nylon surface. Again, compared to the clean area, the LS signal from any greasy print was low while it increased in the presence of a powdered material. Analogous results were obtained on the white varnished substrate, the fluorescence of which was higher than the characteristic one from the examined nitro-compounds placed as fingerprints.

\subsection{Internal Camera}

The internal camera captures the target image magnified through the telescope and its operation could be selected both for checking the surface state (Figure 6) and to point precisely the instrument. The latter capability was exploited to perform remote LIBS measurement exactly on the selected small features of the target [11]. 

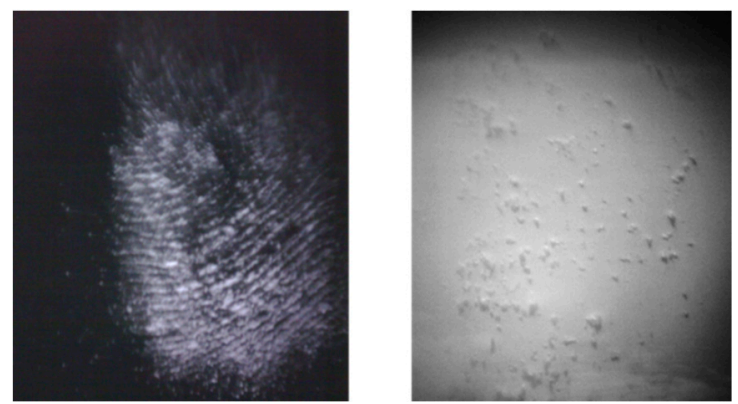

Figure 6. Traces of RDX left by a fingerprint and observed by the internal camera at a target distance of $10 \mathrm{~m}$ : left, on black polypropylene; right, on white varnished metal.

\subsection{Raman}

At a target distance of $10 \mathrm{~m}$, by applying the laser energy of $16 \mathrm{~mJ}$ and the signal accumulation over 50 laser shots, we observed characteristic Raman peaks from the first 10 fingerprint generations containing any of the examined nitro-compounds except TNT. Figure 7, left, shows the raw Raman spectra detected from RDX fingerprints placed on the white varnished metal. The corresponding processed spectra and the RDX peaks detected automatically are depicted on the right panel. On the first-generation fingerprint, with the estimated RDX mass in the order of $1 \mathrm{mg}$, six characteristics peaks were detected (Figure 7d). On the third fingerprint, four peaks were assigned automatically (Figure 7e) while on the ninth-generation fingerprint only the two most intense Raman lines emerged from the noise. On the RDX fingerprint of the tenth generation (not shown) the only recognized peak was centered on $885 \mathrm{~cm}^{-1}$, corresponding to $\mathrm{C}-\mathrm{N}-\mathrm{C}$ symmetric ring breathing. In all the cases the vibration peak from $\mathrm{O}_{2}\left(1555 \mathrm{~cm}^{-1}\right)$ was present in the spectra. The Raman peaks from RDX were not detected on fingerprints of the 4 th, 6 th, and 7 th generations.

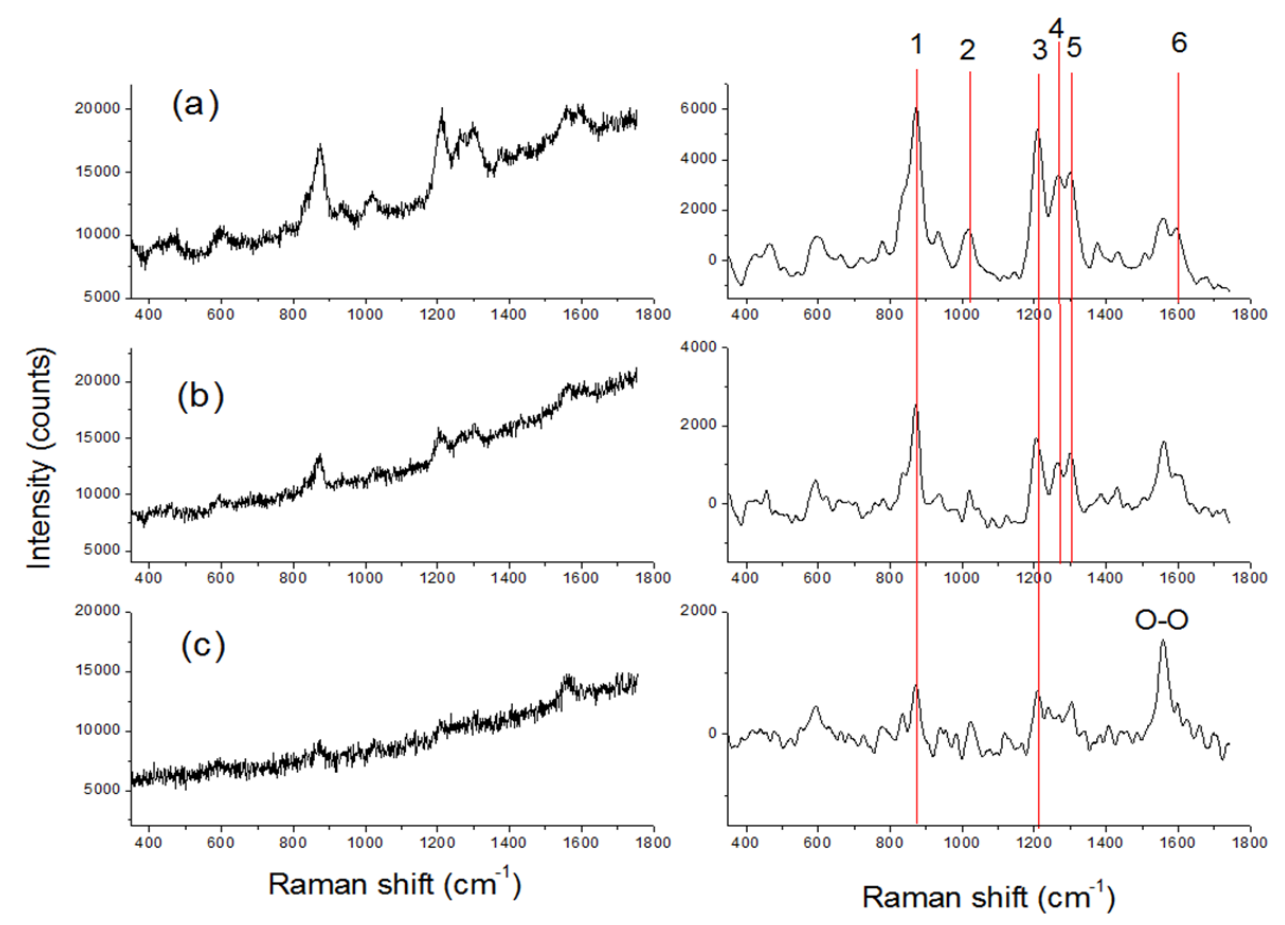

Figure 7. Raw (left) and processed (right) Raman spectra from RDX fingerprints of the 1st (a), 3rd (b), and 9th (c) generation placed on the white varnished metal. Red lines on the left panel indicate the RDX peaks detected automatically by the instrument's software. 
In eye-safe regime we performed Raman measurements on different first-generation fingerprints at a distance of $10 \mathrm{~m}$. Here, we applied a laser fluence of $4.6 \mathrm{~mJ} / \mathrm{cm}^{2}$, slightly lower than the maximum permissible exposure, and a single shot acquisition. Successful Raman detection of ammonium-nitrate, urea-nitrate and RDX in the eye-safe regime was obtained also on fluorescing targets (Figure 8) while no peaks were observed from PETN and TNT.

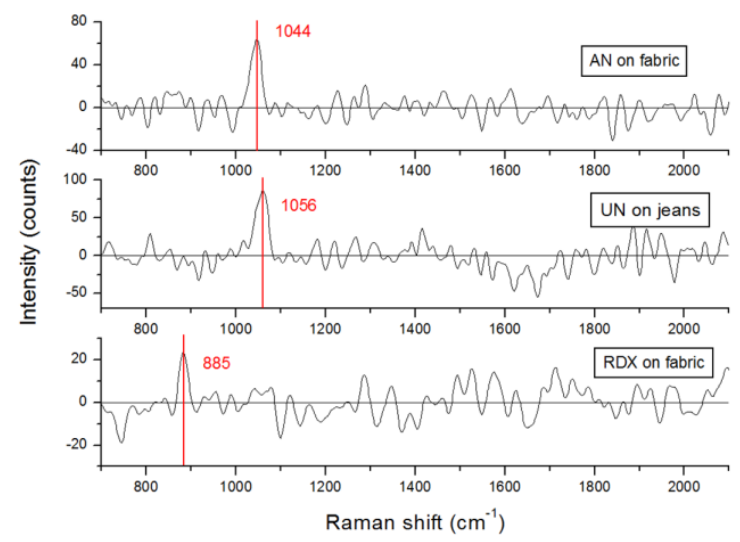

Figure 8. Processed single-shot Raman spectra in eye-safe regime measured on the first-generation fingerprints of ammonium-nitrate, RDX, or urea-nitrate placed on a dyed tissue at a $10 \mathrm{~m}$ distance from the instrument. Red lines indicate the peaks detected automatically by the instrument's software.

Figure 9 compares the captured Raman spectra from Teflon placed at a distance of $10 \mathrm{~m}$ or $30 \mathrm{~m}$ for an incident laser energy of $16 \mathrm{~mJ}$. At the higher distance the laser spot diameter, matching the area collected by the fiber bundle, was almost three-times larger (see Table 2). The corresponding peak intensities were about three-times weaker than at distance of $10 \mathrm{~m}$. These differences could be attributed both to the smaller solid angle for signal collection and to larger radiation losses (scattering and absorption) when increasing the optical path through an air atmosphere. However, the Raman spectra here were acquired for the laser energy that was only $7 \%$ of the available while the energy density, also at a $10 \mathrm{~m}$ distance, was much lower than the threshold for damage of the target material. Consequently, we might conclude that Raman measurements on homogeneous materials could be performed with the same response when extending the detection range to $30 \mathrm{~m}$ and beyond if increasing the laser energy, here available up to $230 \mathrm{~mJ}$.

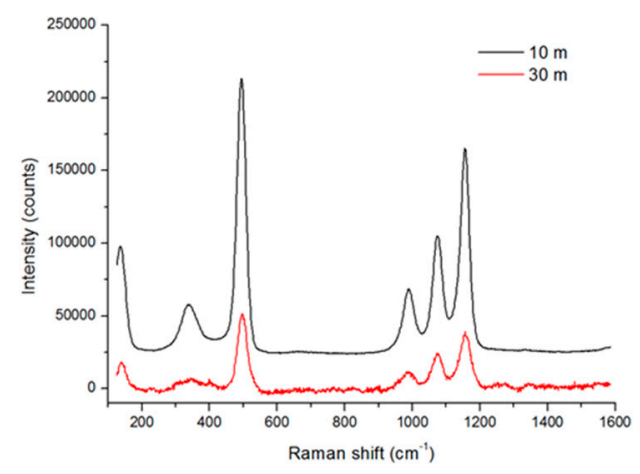

Figure 9. Raman spectrum from Teflon acquired at a target distance of $10 \mathrm{~m}$ (black line) and $30 \mathrm{~m}$ (red line) by accumulating the signal over 50 laser shots with the energy of $16 \mathrm{~mJ}$.

\subsection{LIBS}

At a working distance of $10 \mathrm{~m}$ the LIBS signal was created by pulses with the incident energy of $330 \mathrm{~mJ}$. The spot size on the target was optimized for the maximum plasma emission intensity on the polypropilene sample, and it corresponded to a diameter of $1.3 \mathrm{~mm}$. The spectra were acquired 
with the acquisition gate delay and a gate of $2.5 \mu$ s and $10 \mu \mathrm{s}$, respectively; the ICCD gain was set to 2500. At each sampling point three laser pulses were delivered, registering the spectrum after each shot. Particles around the sampled spot were blasted away by the laser-induced shockwaves, leaving an almost clean area of about $6 \mathrm{~mm}$ in diameter. For this reason, the LIBS sampling was performed at points distanced for at least $4 \mathrm{~mm}$ from each other. On different tested substrates, in the presence of nitro-compound particles the atomic line intensities from $\mathrm{H}, \mathrm{N}$, and $\mathrm{O}$ were higher after the first laser shot than after the second one (Figure 10, right panel) although the C I peak (not shown) and the UV $\mathrm{CN}$ lines (Figure 10, left panel) might have similar intensities in the both cases. This is particularly evident on substrates with a low ablation rate, as for example textiles (fabric, jeans). Differently, the areas contaminated by greasy residues showed a significantly reduced overall plasma intensity compared to the bare material. We exploited the differences in the spectra after the first laser shot, interacting with the surface residue, and the second shot, ablating the cleaned substrate, to achieve identification of the nitro-compounds also on organic substrates.
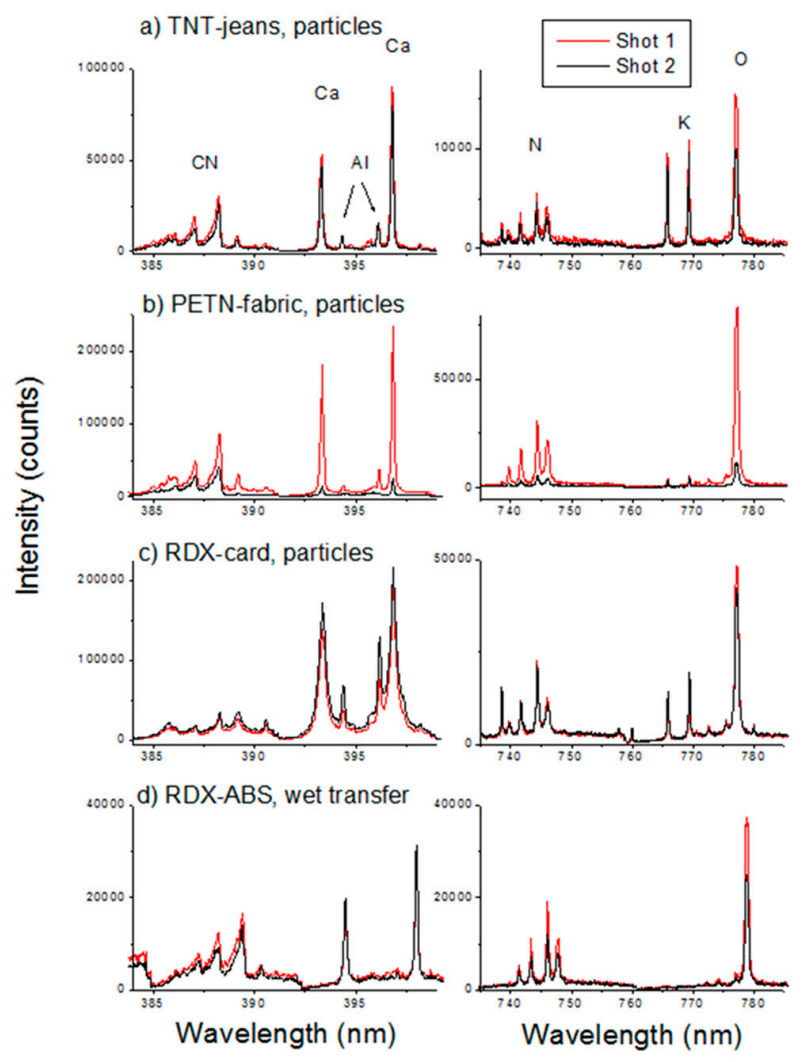

Figure 10. Single-shot LIBS spectra in intervals containing CN molecular features (left panel), $\mathrm{N}$ I and O I emission (right panel), for explosives in the form of particles $(\mathbf{a}-\mathbf{c})$ or delivered by a wet transfer $(\mathbf{d})$ on different organic substrates.

Figure 11 shows the sum $\mathrm{S}$ of the ratio $\mathrm{R}(\mathrm{H})$ relative to $\mathrm{H} \alpha$ line peak after the first and the second laser shot and of the analogue ratio $\mathrm{R}(\mathrm{N})$ relative to the $\mathrm{N} I$ line $(746.83 \mathrm{~nm})$. We excluded the $\mathrm{O} I$ line from in the nitro-compound's identification because some substrates might have surface oxidation, which could increase the intensity of this line after the first laser pulse compared to the second one. On the black polypropylene (Figure 11, top) the clean material and the same with dust particles had a very similar range of the sum $S=R(H)+R(N)$ while the $S$ value was significantly lower in presence of greasy residues. On the other hand, the sum $S$ increases up to eight times when passing from the clean polypropylene to areas containing particles of ammonium-nitrate, PETN, or RDX (the only nitro-compounds tested on this substrate). Similar results were obtained on the white varnished metal where also TNT and urea-nitrate were tested. 


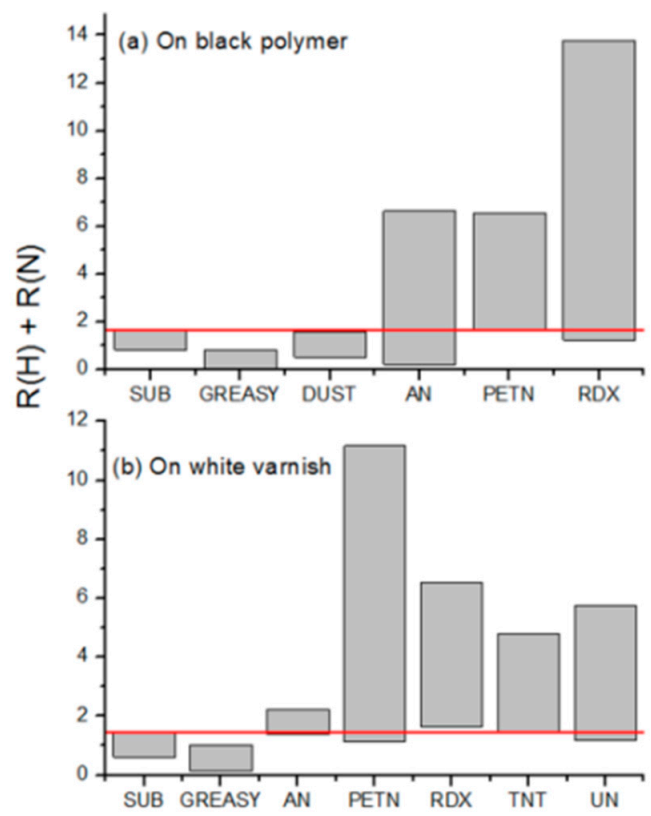

Figure 11. Span of the LIBS measured sum $S$ of the ratios $R(H)=I_{1}(H) / I_{2}(H)$ and $R(N)=I_{1}(N) / I_{2}(N)$ on different fingerprints placed over black polypropylene (a) or white varnished metal (b). The red line indicates the lower threshold established for the detection of nitro-compounds. X axis: SUB is clean substrate, $\mathrm{AN}$ is ammonium-nitrate and $\mathrm{UN}$ is urea-nitrate.

In the ILS system, presently, the algorithm for the automatic recognition of nitro-compounds by LIBS is based on determining the sum $\mathrm{S}$ and assigning the hazard on the target's cell if $\mathrm{S}>1.6$.

At a target distance of $30 \mathrm{~m}$, the shortest wavelength of the lines detected by LIBS with the pulse energy of $330 \mathrm{~mJ}$ was around $227 \mathrm{~nm}$, and this corresponded to $\mathrm{Al}$ I transitions from aluminum substrate. It was not possible to compare directly the LIBS spectra for distances of $30 \mathrm{~m}$ and $10 \mathrm{~m}$ because the optimized acquisition parameters were different in the two cases. However, at $30 \mathrm{~m}$ and in a single shot acquisition it was possible to observe, besides the lines from $\mathrm{Al}$, also a number of impurities, as for example $\mathrm{Mg}, \mathrm{Ca}, \mathrm{Ti}, \mathrm{Si}, \mathrm{Na}$, and $\mathrm{K}$. The analogue probing of the Teflon sample (Figure 12, top) revealed the C I line at $247.8 \mathrm{~nm}$ and different impurities.

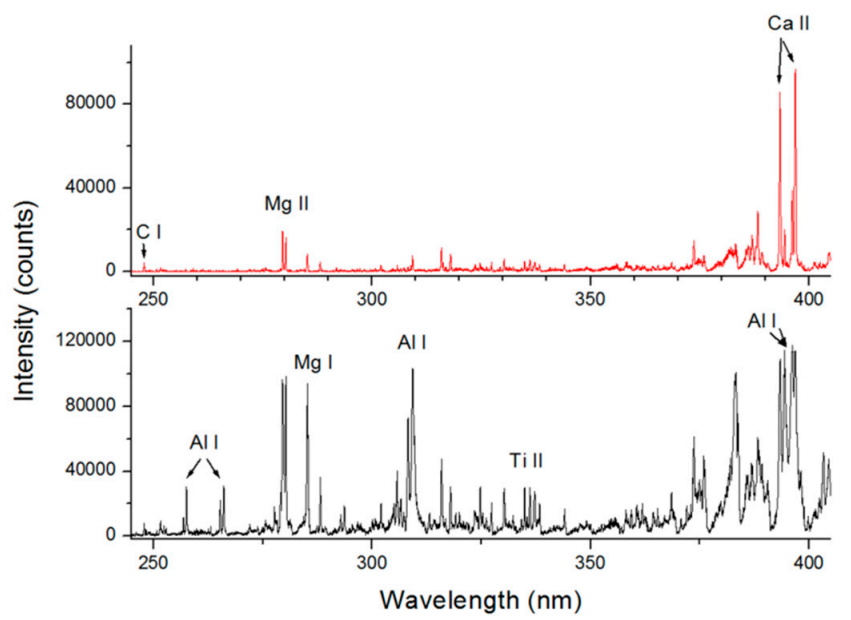

Figure 12. UV portion of a single-shot LIBS spectrum acquired at distance of $30 \mathrm{~m}$ on: top, Teflon substrate; bottom, aluminum target. 


\section{Discussion}

It is well known that the number of particles and their distribution vary significantly from one fingerprint to successive prints [28,29], where the transfer efficiency also depends on the adhesive properties of the substrate [30]. For example, the first-generation fingerprint containing RDX particles might be heavily loaded, with a mass in the order of $1 \mathrm{mg}$, while already the fifth fingerprint contained a particle mass 1000-times lower [15]. The first prints rapidly loose the large-diameter heavy particles and then the transfer efficiency to the next prints, containing mainly small and light particles, is high. For example, in [29], it was found that the RDX mass measured for the fifth fingerprint was of $1.7 \mu \mathrm{g}$, while the 50th fingerprint had a mass of $0.23 \mu \mathrm{g}$. Furthermore, the prints did not exhibit a gradual progression from the heaviest loading to the lightest, and this might explain why we missed detecting RDX by Raman technique on some fingerprints, but recovered the signal at successive print generations.

To estimate roughly the detection sensitivity of the ILS techniques, we started from the typical RDX particle mass per unit area in fingerprints, based on the data reported in [29]. We encircled the area of the shown fingerprint on the dimensionally-calibrated photo of $29 \times 18 \mathrm{~mm}$ and found that the print occupied about $4.1 \mathrm{~cm}^{2}$. The extrapolated particle mass of RDX inside the 10th fingerprint was about $1 \mu \mathrm{g}$, corresponding to the mean mass density of $\mathrm{MD}_{10}=0.24 \mu \mathrm{g} / \mathrm{cm}^{2}$.

The LS-LIF signals at a distance of $10 \mathrm{~m}$ were collected from a spot diameter of $3.3 \mathrm{~mm}$ (area $0.085 \mathrm{~cm}^{2}$ ). On a non-fluorescing or weakly-fluorescing substrate, all the examined nitro-compounds excluding PETN showed their own LIF spectrum, detectable up to the 10th fingerprint, which corresponded to the mean probed particle mass of $20 \mathrm{ng}$. Furthermore, the LIF signal intensity might be improved by increasing the laser fluence inside the target area captured by the optical collection system. This could be achieved both by focusing the high-power laser beam more tightly down to a diameter of 3.3. $\mathrm{mm}$ or by increasing the laser energy from $16 \mathrm{~mJ}$, used here, to, for example, $200 \mathrm{~mJ}$. Raising the laser energy to $200 \mathrm{~mJ}$ in the same focusing conditions would lead to an energy density of $0.30 \mathrm{~J} / \mathrm{cm}^{2}$, too low to induce ablation on most of the materials. In the case of RDX, the lower damage threshold under 355-nm irradiation by ns pulses is about $1.1 \mathrm{~J} / \mathrm{cm}^{2}$ [31]. However, when working on the black polypropilene substrate we noticed some sound released during the beam-target interaction for laser energies above $60 \mathrm{~mJ}$, caused by rapid surface heating although the substrate remained unaltered under visual inspection. Thus, in order to avoid thermal effects on any target, in the systematic measurements we limited the UV laser energy. From the previous considerations, we might conclude that for the fluorescing nitro-compounds on a non-fluorescing substrate placed at a 10-m distance, the particles might be detected by the LIF technique (of the ILS system) down to 10 ng without altering the target. This sensitivity is sufficient to observe high-generation fingerprints although the LIF technique, itself, cannot be considered discriminatory for nitro-compounds. On the other hand, the examined liquid organic residues were highly fluorescing, but with the emission peak close to the laser excitation wavelength, and they might be detected in much smaller quantities than the nitro-compounds.

The detection sensitivity analogue to the LIF might be deduced also for the LS measurements on dark substrates. Here, the back-scattered signal from the continuous-wave red laser could be increased by applying longer acquisition times. While surface particles increased the LS signal compared to the dark substrate, the presence of greasy residues heavily lowered the backscattered signal, if present on the bare substrate. In this way, the combination of LS-LIF measurements was extremely useful for tagging the target areas containing some surface contaminants, supplying also information about the residue state: particles or liquid/gel.

In the case of a highly fluorescing and scattering substrates like the examined white varnished metal, the presence of residues might be deduced from changes in the LS-LIF spectral intensity and distribution (for LIF). In such a case, the sensitivity, both of the LIF and the LS measurements, is more difficult to estimate because the detection of residues is based on differences in the response between the bare and the contaminated area, and not on the absolute signal intensities. 
Regarding the Raman measurements, the considerations were analogous to the LIF technique, but in this case the signal was collected from a larger area, corresponding to $0.66 \mathrm{~cm}^{2}$. Assuming the presence of $1 \mu \mathrm{g}$ of a nitro-compound in the 10th-generation fingerprints, the Raman signals of which were weak but generally observable (excluding TNT), the instrument's detection sensitivity at a 10-m distance was of about $150 \mathrm{ng}$. This detection limit could be further improved by increasing the energy of the high-power laser a few times in the absence of an intense substrate fluorescence that could mask the weak Raman signal.

In the LIBS measurements at a distance of $10 \mathrm{~m}$, the energy density on the target was $24.9 \mathrm{~J} / \mathrm{cm}^{2}$. This laser fluence was sufficient to create plasma on all the tested samples and it was well above the $100 \%$ ignition threshold for RDX [31] and PETN [32] under the analogue laser excitation. On residues of these secondary explosives the measured sum $S$ achieved values $>11$, more than six-times higher than those measured on the bare substrate. A few random missing detection points $(S<1.6)$ for RDX on the black polypropylene and for PETN on the white varnish could be attributed to a locally low particle presence inside the small laser spot. TNT was tested only on the white varnish and it was always detected by LIBS, although with $S$ values $<5$. Given that this residue was not observed by Raman, we believe that its presence on the target was lower than expected because of its relatively easy evaporation.

Ammonium-nitrate and urea-nitrate, which are not explosives but oxidizers, are less sensitive to shock than the explosives. For these nitrates the determined $S$ values were always $<7$. In particular, very low $S$ values in some prints of ammonium-nitrate compared to the bare black substrate might be attribute to the hygroscopic properties of this substance, where the presence of the absorbed water highly reduced the plasma emission intensity. Beside the very low plasma emission intensity when the substrates were covered by liquid/gel residues, it is interesting to note that in the presence of dust contamination, the $S$ values were equal to or smaller than on the clean substrate. This indicates that the increased LIBS intensities of the H I and N I (the elements also present in air) lines on areas containing explosives or oxidizers were not caused by an improved laser-particle interaction compared to the laser-substrate, but to the plasma temperature increase due to the laser-induced micro-explosions and endothermic reactions [31-33]

Considering that the laser spot in the LIBS measurements was of only $1.3 \mathrm{~mm}$ in diameter and assuming an uniform particle distribution, the 10th-generation fingerprint would contain about $3 \mathrm{ng}$ of particles inside the probed spot. This mass could be approximately considered as the detection limit reached on the tested samples. In the future, the effect of higher laser energy density on the detection sensitivity by LIBS could be examined, but taking into account that a higher incident energy might increase the contribution of the target to the plasma produced on a surface residue.

The herein applied approach for the detection of nitro-compounds by LIBS, based on the spectral differences after the first and the second laser pulse at the same position, was much simpler and straightforward than the methods proposed in [15,33-36]. Our approach was not affected by missing plasma stoichiometry [36] and it reduced the influence of substrate material on the classification results. Furthermore, this method exploited only the emission lines in the red-NIR spectral region, which are less sensitive to the atmospheric attenuation than UV spectral features, and this is a promising factor for the stand-off LIBS detection of trace explosives at longer distances than reported in this work.

\section{Conclusions}

The herein described ILS system allowed scanning of remote targets, producing 2D thematic maps. By simultaneous LS and LIF and measurements it was possible to observe changes of the material from one point to another, to identify areas with the presence of surface residues and to have indications if the surface contaminants were liquid or in the form of particles. Visualization of the surface details was also possible by the internal camera, which works with a high magnification optical system. Raman probing allowed recognizing the molecular composition of residues (if present in a sufficient quantity) and tagging the areas where nitro-compounds were identified. Similarly, target scanning 
by LIBS tagged areas containing a generic nitro-compound. The estimated detection sensitivities for nitro-compound particles for a target distance of $10 \mathrm{~m}$ were in the order of $10 \mathrm{ng}, 100 \mathrm{ng}$, and $1 \mathrm{ng}$ for LS-LIF, Raman, and LIBS, respectively.

The ILS instrument is fully automatized, equipped with a user-friendly software interface controlled via wireless communication. These characteristics permits operating also in hazardous conditions, either from a fixed position or mounting the system on a robotic vehicle. Except for LIBS, the measurements could be performed also in eye-safe regime, but with reduced detection sensitivity. The tests were performed up to a distance of $30 \mathrm{~m}$ and using less than $10 \%$ or $40 \%$ of the available laser energy at $355 \mathrm{~nm}$ and $1064 \mathrm{~nm}$, respectively. Consequently, it could be expected that the instrument's maximum working distance might largely exceed $50 \mathrm{~m}$.

In this work we focused on the instrument performances and its ability to detect specifically the nitro-compounds. Nevertheless, by recalling different data processing algorithms, the ILS could produce many other thematic maps, both molecular and elemental, among them for example: recognizing the polymer type or producing a distribution of heavy elements inside the scanned area.

The ILS is a highly-performing scientific instrument and it is intended as a starting point for the development of other simplified instruments for some specific applications, such as characterization of cultural heritage objects, forensic, environmental monitoring and many others.

\section{Patents}

V. Lazic, A. Palucci, L. De Dominicis, M. Nuvoli, M. Pistilli, I. Menicucci, F. Colao, S. Almaviva, Dispositivo ILS (Integrated Laser Sensor) per le analisi di materiali con tecniche Raman, LIF (Laser Induced Fluorescence) e LIBS (Laser Induced Breakdown Spectroscopy). Patent deposited 28 December 2017 at Ministero dello Sviluppo Economico, Italy, Number 102017000150309.

Author Contributions: V.L.: the leader in the conceptual design, projecting, realization, testing of the ILS, and in scientific works involving the instrument; A.P.: conceptual design, coordination, and funding acquisition; L.D.D.: project reporting and organization of the first outdoor campaign; M.N., design of the mechanical parts and trials; M.P., the instrument assembling and trials; I.M., design and realization of electronic controls and trials; F.C.: communication protocols and software; S.A.: ILS characterization and trials.

Funding: This work was supported by the FP7 project EDEN, EC Grant 313077.

Conflicts of Interest: The authors declare no conflict of interest.

\section{References}

1. Acosta-Maeda, T.E.; Misra, A.; Muzangwa, L.G.; Berlanga, G.; Muchow, D.; Porter, J.; Sharma, S.K. Remote Raman measurements of minerals, organics, and inorganics at $430 \mathrm{~m}$ range. Appl. Opt. 2016, 55, 10283-10289. [CrossRef] [PubMed]

2. Chirico, R.; Almaviva, S.; Colao, F.; Fiorani, L.; Nuvoli, M.; Schweikert, W.; Schnürer, F.; Cassioli, L.; Grossi, S.; Murra, D.; et al. Proximal Detection of Traces of Energetic Materials with an Eye-Safe UV Raman Prototype Developed for Civil Applications. Sensors 2016, 16, 8. [CrossRef] [PubMed]

3. Gaft, M.; Nagli, L. UV gated Raman spectroscopy for standoff detection of explosives. Opt. Mater. 2018, 30, $1739-1746$. [CrossRef]

4. Sharma, S.K.; Misra, A.K.; Sharma, B. Portable remote Raman system for monitoring hydrocarbon, gas hydrates and explosives in the environment. Spectrochim. Acta Part A 2005, 61, 2404-2412. [CrossRef]

5. Sharma, S.K. New trends in telescopic remote Raman spectroscopic instrumentation. Spectrochim. Acta Part A 2007, 68, 1008-1022. [CrossRef] [PubMed]

6. Spizzichino, V.; Caneve, L.; Colao, F.; Ruggiero, L. Characterization and Discrimination of Plastic Materials Using Laser-Induced Fluorescence. Appl. Spectrosc. 2016, 70, 1001-1008. [CrossRef]

7. Kumar, D.; Sharma, R.C.; Maini, A.K. Standoff Laser-Induced Fluorescence Sensors for Biological Warfare Agents. Spectrosc. Lett. 2013, 46, 147-153. [CrossRef]

8. Caneve, L.; Colao, F.; Fantoni, R.; Fiorani, L. Scanning lidar fluorosensor for remote diagnostic of surfaces. Nucl. Instrum. Methods Phys. Res. A 2013, 720, 164-167. [CrossRef] 
9. Matvienko, G.G.; Grishin, A.I.; Kharchenko, O.V.; Romanovskii, O.A. Application of laser-induced fluorescence for remote sensing of vegetation. Opt. Eng. 2006, 45, 056201. [CrossRef]

10. Álvarez-Trujillo, L.A.; Lazic, V.; Moros, J.; Laserna, J.J. Standoff monitoring of aqueous aerosols using nanosecond laser-induced breakdown spectroscopy: Droplet size and matrix effects. Appl. Opt. 2017, 56, 3773-3782. [CrossRef]

11. Lazic, V.; Trujillo-Vazquez, A.; Sobral, H.; Márquez, C.; Palucci, A.; Ciaffi, M.; Pistilli, M. Corrections for variable plasma parameters in laser induced breakdown spectroscopy: Application on archeological samples. Spectrochim. Acta Part B 2016, 122, 103-113. [CrossRef]

12. Kumar, S.; Vasa, N.J.; Sarathi, R. Remote surface pollutant measurement by adopting a variable stand-off distance based laser induced spectroscopy technique. J. Phys. D Appl. Phys. 2015, 48, 435504. [CrossRef]

13. Gaona, I.; Lucena, P.; Moros, J.; Fortes, F.J.; Guirado, S.; Serrano, J.; Laserna, J.J. Evaluating the use of standoff LIBS in architectural heritage: Surveying the Cathedral of Màlaga. J. Anal. At. Spectrom. 2013, 28, 810-820. [CrossRef]

14. Vítková, G.; Novotný, K.; Prokeš, L.; Hrdlička, A.; Kaiser, J.; Novotný, J.; Malina, R.; Prochazka, D. Fast identification of biominerals by means of stand-off laser-induced breakdown spectroscopy using linear discriminant analysis and artificial neural networks. Spectrochim. Acta Part B 2012, 73, 1-6. [CrossRef]

15. Lòpez-Moreno, C.; Palanco, S.; Laserna, J.J.; DeLucia, F., Jr.; Miziolek, A.W.; Rose, J.; Walters, R.A.; Whitehouse, A.I. Test of a stand-off laser-induced breakdown spectroscopy sensor for the detection of explosive residues on solid surfaces. J. Anal. At. Spectrom. 2006, 21, 55-60. [CrossRef]

16. López-Moreno, C.; Palanco, S.; Laserna, J.J. Stand-off analysis of moving targets using laser-induced breakdown spectroscopy. J. Anal. At. Spectrom. 2007, 22, 84-87. [CrossRef]

17. Wiens, R.C.; Sharma, S.K.; Thompson, J.; Misra, A.; Lucey, P.G. Joint analyses by laser-induced breakdown spectroscopy (LIBS) and Raman spectroscopy at stand-off distances. Spectrochim. Acta Part A 2005, 61, 2324-2334. [CrossRef]

18. Moros, J.; Lorenzo, J.A.; Lucena, P.; Tobaria, L.M.; Laserna, J. Simultaneous Raman spectroscopy-laser-induced breakdown spectroscopy for instant standoff analysis of explosives using a mobile integrated sensor platform. Anal. Chem. 2010, 82, 1389-1400. [CrossRef] [PubMed]

19. Lin, Q.; Niu, G.; Wang, Q.; Yu, Q.; Duan, Y. Combined Laser-Induced Breakdown with Raman Spectroscopy: Historical Technology Development and Recent Applications. Appl. Spectrosc. Rev. 2013, 48, 487-508. [CrossRef]

20. Lednev, V.N.; Pershin, S.M.; Sdvizhenskii, P.A.; Grishin, M.Y.; Fedorov, A.N.; Bukin, V.V.; Shchegolikhin, A.N. Combining Raman and laser induced breakdown spectroscopy by double pulse lasing. Anal. Bioanal. Chem. 2018, 410, 277-286. [CrossRef]

21. Osticioli, I.; Mendes, N.F.C.; Nevin, A.; Zoppi, A.; Lofrumento, C.; Becucci, M.; Castellucci, E.M. A new compact instrument for Raman, laser-induced breakdown, and laser-induced fluorescence spectroscopy of works of art and their constituent materials. Rev. Sci. Instrum. 2009, 80, 076109. [CrossRef] [PubMed]

22. Abedin, M.N.; Bradley, A.T.; Sharma, S.K.; Misra, A.K.; Lucey, P.G.; McKay, C.P.; Ismail, S.; Sandford, S.P. Mineralogy and astrobiology detection using laser remote sensing instrument. Appl. Opt. 2015, 54, 7598-7611. [CrossRef] [PubMed]

23. Perez, R.; Parès, L.P.; Newell, R.; Robinson, S. The Supercam instrument on the NASA Mars 2020 mission: Optical design and performance. Proc. SPIE 2016, 10562, 105622K.

24. Wiens, R.C.; Maurice, S.; Perez, F.R. The SuperCam Remote Sensing Instrument Suite for the Mars 2020 Rover: A Preview. Spectroscopy 2017, 32, 50-55.

25. Wallin, S.; Pettersson, A.; Östmark, H.; Hobro, A. Laser-based standoff detection of explosives: A critical review. Anal. Bioanal. Chem. 2009, 395, 259-274. [CrossRef] [PubMed]

26. Skvortsov, L.A. Laser methods for detecting explosive residues on surfaces of distant objects. Quantum Electron. 2012, 42, 1-11. [CrossRef]

27. Hallowell, S.F. Screening people for illicit substances: A survey of current portal technology. Talanta 2001, 54, 447-458. [CrossRef]

28. Gresham, G.L.; Davies, J.P.; Goodrich, L.D.; Blackwood, L.G.; Liu, B.Y.H.; Thimsem, D.; Yoo, S.H.; Hallowel, S.F. Development of particle standards for testing detection systems: Mass of RDX and particle size distribution of composition 4 residues. Proc. SPIE 1994, 2275, 34-44.

29. Verkouteren, J.R.; Coleman, J.L.; Cho, I. Automated Mapping of Explosives Particles in Composition C-4 Fingerprints. J. Forensic Sci. 2010, 55, 334-340. [CrossRef] 
30. Lees, H.; Zapata, F.; Vaher, M.; García-Ruiz, C. Study of the adhesion of explosive residues to the finger and transfer to clothing and luggage. Sci. Justice 2018, 58, 415-424. [CrossRef]

31. Yan, Z.; Zhang, C.; Liu, W.; Li, J.; Huang, M.; Wang, X.; Zhou, G.; Tan, B.; Yang, Z.; Li, Z.; et al. Ultraviolet Laser-induced ignition of RDX single crystal. Sci. Rep. 2016, 6, 20251. [CrossRef] [PubMed]

32. Kalenskii, A.V.; Zvekov, A.A.; Galkina, E.V. The critical parameters of the thermal explosion micro hotspot model dependence on the pulse duration. IOP Conf. Ser. J. Phys. 2017, 830, 012128. [CrossRef]

33. Gottfried, J.L.; De Lucia Jr, F.C.; Munson, C.A.; Miziolek, A.W. Strategies for residue explosives detection using laser-induced breakdown spectroscopy. J. Anal. At. Spectrom. 2008, 23, 205-216. [CrossRef]

34. Shaik, A.K.; Epuru, N.R.; Syed, H.; Byram, C.; Soma, V.R. Femtosecond laser induced breakdown spectroscopy based standoff detection of explosives and discrimination using principal component analysis. Opt. Express 2018, 26, 8069-8083. [CrossRef] [PubMed]

35. Lazic, V.; Palucci, A.; Jovicevic, S.; Poggi, C.; Buono, E. Analysis of explosive and other organic residues by laser induced breakdown spectroscopy. Spectrochim. Acta Part B 2009, 64, 1028-1039. [CrossRef]

36. Lazic, V.; Palucci, A.; Jovicevic, S.; Carpanese, M. Detection of explosives in traces by laser induced breakdown spectroscopy: Differences from organic interferents and conditions for a correct classification. Spectrochim. Acta Part B 2011, 66, 644-655. [CrossRef]

(C) 2019 by the authors. Licensee MDPI, Basel, Switzerland. This article is an open access article distributed under the terms and conditions of the Creative Commons Attribution (CC BY) license (http://creativecommons.org/licenses/by/4.0/). 